\section{H-Comp: A program to calculate information complexity}

\author{
MICHAEL E. NIELSEN \\ Georgia Southern University, Statesboro, Georgia
}

Scott's (1969) H is a statistic that represents the number of independent dimensions in a set of binary $(1,0)$ data. $\mathrm{H}$ has been used by personality and social psychologists to measure the complexity of thought people have about themselves (self-complexity) and about other people. This article describes $\mathrm{H}-\mathrm{Comp}$, a program that calculates $\mathrm{H}$. Using data input from either the keyboard or from an ASCII data file, $\mathrm{H}-\mathrm{Comp}$ calculates $\mathrm{H}$ and writes the resulting score and raw data to output files. $\mathrm{H}-\mathrm{Comp}$ provides an accurate and efficient way to calculate $\mathrm{H}$.

$H$ is a measure of the redundancy contained in sets of information in binary $(1,0)$ data strings (Scott, 1969). Developed over 30 years ago by information theorists (Attneave, 1959), $H$ represents the number of independent dimensions that a person uses to describe something. Greater complexity is apparent when the person views the object as having many different aspects and makes fine distinctions among those aspects. Complexity is lower when the person considers the object as having fewer aspects, or when the person describes those aspects in relatively redundant terms.

Psychologists have begun to use $H$ to assess an individual's complexity of thought in two general lines of research. In the first, researchers examine the complexity of thought regarding the members of various groups (Linville, 1982; Linville, Fischer, \& Salovey, 1986; Linville \& Jones, 1980). Results in this area indicate that people describe ingroups-those groups to which they belong - as having many different types of people or characteristics. On the other hand, people describe outgroups - those groups to which they do not belong-as being relatively homogeneous. Consequently, members of outgroups are stereotyped as having certain general characteristics. This "outgroup homogeneity" effect suggests that a person possesses stereotypes, in part, because of a limited set of information regarding the membership of the outgroup.

The second line of research using $H$ focuses on selfcomplexity, or the complexity of thoughts that one has about oneself (Dixon \& Baumeister, 1991; Hershberger, 1990; Kalthoff \& Niemeyer, 1993; Linville, 1985, 1987; Niedenthal, Setterlund, \& Wherry, 1992; Nielsen, 1992; Showers, 1992; Smith \& Cohen, 1993). Individuals with greater self-complexity use different adjectives to describe themselves in their many social roles, whereas people with less self-complexity tend to use the same

Correspondence should be addressed to M. E. Nielsen, Department of Psychology, Georgia Southern University, Statesboro, GA 304608041 (e-mail:mnielsen@gasou.edu). terms repetitiously in describing their fewer social roles (Linville, 1985). In other words, complex people have a greater number of aspects to their life, and they make many fine distinctions between those aspects.

$H$ has also played an important role in studies of people's reactions to stressful events. People with greater self-complexity are more resistant than people with less self-complexity to the effects of stress (Linville, 1985, 1987). For example, greater self-complexity acts as a buffer against stress-related illness (Linville, 1987). The buffering effect measured by $H$ appears to be unique; other measures of complex thought fail to reveal the buffering effect that $H$ demonstrates (Kalthoff $\&$ Neimeyer, 1993). $H$ also yields a more accurate estimate of the buffering effect than does a simple count of a person's social roles (Linville, 1987).

\section{Calculating $\boldsymbol{H}$}

In a typical self-complexity study, a person is asked to think of himself or herself while sorting a set of adjective cards into meaningful units. One adjective is written on each card; the cards may be used more than once, and can be placed in any number of combinations. For instance, suppose that a person sorts a set of 33 cards into three self-aspects. The first aspect, which is labeled "Student," includes the adjectives honest, intelligent, organized, confident, alert, happy, outgoing, and conscientious. The second aspect, labeled "Worker," includes honest, conscientious, happy, helpful, and tired. The third aspect, labeled "Sorority Sister," includes the adjective happy, conscientious, outgoing, sociable, and attractive. $H$ then would be applied to this information to arrive at a measure of the redundancy of these self-descriptions. $H$ would be calculated in three steps.

1. Each self-aspect is relabeled; Student is labeled "A," Worker is labeled " $\mathrm{B}$," and Sorority Sister is labeled "C."

2. Adjectives are grouped into sets that are used similarly. In this example, Group A includes only those adjectives that were used in describing the first aspect, $B$ the second aspect, and so on. In the present example, the adjectives are sorted as follows:
A: intelligent, organized, confident, alert
B: helpful, tired
C: sociable, attractive
$\mathrm{AB}$ : honest
AC: outgoing
ABC: happy, conscientious

3. These adjective sorts are applied to the $H$ formula (Scott, 1969):

$$
H=\log _{2} n-(1 / n)\left(\sum_{i} n_{i} \log _{2} n_{i}\right),
$$

where $n$ is the total number of attributes, and $n_{i}$ is the number of attributes that appear in a particular combination in the descriptions of self-aspects. Higher scores indicate greater complexity. In this case, 


$$
\begin{aligned}
H= & 5.04439-(1 / 33)\left[(21)\left(\log _{2} 21\right)+\right. \\
& (4)\left(\log _{2} 4\right)+(2)\left(\log _{2} 2\right)+(2)\left(\log _{2} 2\right)+ \\
& \left.(1)\left(\log _{2} 1\right)+(1)\left(\log _{2} 1\right)+(2)\left(\log _{2} 2\right)\right] \\
= & 5.04439-(.030303)(106.23872) \\
= & 1.82504 .
\end{aligned}
$$

Note that the quantity $(21)\left(\log _{2} 21\right)$ comes from the 21 adjectives that were available for use, but went unused. Likewise, the quantity $(4)\left(\log _{2} 4\right)$ is from the four adjectives (intelligent, organized, confident, and alert) used only to describe Aspect A, and so on. Note also that, although happy and conscientious may connote different constructs, they are treated by $H$ as a single independent dimension because the subject uses them in the same way.

Because commonly available computer programs do not have routines to calculate $H$, hand calculations are used. Such calculations must be done with great care, however, as they can become tedious and may be prone to errors. Computation of $H$ is facilitated by using a computer to perform the various sorts, comparisons, and calculations. The present program describes such a routine written in PowerBASIC.

Prior to calculating $H$, whether manually or with $H$ Comp, data must be arranged appropriately. This must begin during data collection. For example, Linville (1985, 1987) numbers the adjective cards, and, after the participant completes a sort, records the card numbers used in that particular sort. Linville (personal communication, November 4, 1994) makes several additional recommendations that facilitate the data collection process, help assure that reliable data are collected, and provide a convenient record for the later calculation of $H$. These include (1) pretesting traits before using them with a specific population; (2) having subjects perform the task individually, rather than in groups; and (3) giving subjects complete, unabbreviated instructions for completing the task, such as those found in Linville (1987). ${ }^{1}$

\section{Program Description}

Data can be entered directly at the keyboard or from an ASCII file. If the user selects keyboard entry, $H$ Comp prompts for a subject identifier (ID number or name), the number of adjectives available for the subject to sort, and the number of sorts (self-aspects) to be entered for the subject. Next, the user describes the sorts. An adjective is represented by a " 1 " if it was used in a given sort or by a " 0 " if it was not. $H$-Comp reminds the user that, in order for the resulting calculation to be valid, each adjective must appear in the same position in each sort. For example, if the word calm is written on card number 14, it must always be represented by the 14th digit in each sort entered on the keyboard. If this instruction is not followed, the resulting computation will be invalid.

Data entered at the keyboard are first screened for errors (entries other than 1 and 0 , as well as too many or too few entries). After calculations have been com- pleted, $H$ scores are written to a file called H.DAT. Also written to H.DAT are the subject identifier and the number of sorts created by the participant, which has been used to demonstrate the incremental validity of selfcomplexity over a simple count of self-aspects or social roles (Linville, 1987). The raw data are written to a second file, RAW.DAT, allowing the user to confirm data entry accuracy and to examine the frequency with which specific adjectives were used (Nielsen, 1992). The output files are written to the drive and directory in which $H$-Comp is located.

Data may also be read from an ASCII file, sometimes called a "text" or "nondocument" file, which is created easily with most word processors. If data are in an ASCII file, $H$-Comp prompts for the file name, retrieves the data from the file, and calculates $H$ for each subject in the file. Data in the ASCII file must be formatted in the following fashion. The first line of the file should contain only the first subject's ID number or name. The second line contains the number of adjectives that were available for the subject to sort. The third line contains the number of sorts performed by the subject. Finally, the remaining line(s) each contain a single sort, with a 1 indicating that the adjective was used in that sort, and a 0 indicating that it was not used. For example, suppose that Subject 1 sorted 10 adjectives into three sorts (or selfaspects). In that case, the lines for Subject 1 would be:

\section{Subject 1 \\ 10 \\ 3 \\ 1111101010 \\ 1111100000 \\ 1011000000}

The next line of the ASCII file would begin the listing for the next subject, starting with the subject identification number and continuing with the number of adjectives available, the number of sorts performed, and finally, the specific sorts generated by the subject. As with data entry on the keyboard, each adjective must appear in the same serial position in each sort.

In order to calculate $H, H$-Comp uses the following algorithm.

1. Each adjective sort is treated as a string variable.

2. When all sorts have been read, an additional set of string variables is created. The newly created variables represent the pattern of usage of each adjective across the sorts created by the subject. For instance, if we use the example of our Subject 1 above, the 10 adjectives were sorted three times:

Sort 1: 1111101010

Sort 2: 1111100000

Sort 3: 1011000000

$\mathrm{H}$-Comp creates 10 new variables, representing the pattern of use of each adjective across the three sorts. So, the first variable would be 111 , the second 110 , the third 111 , and so on. 
3. These variables are compared with one another in a loop in order to identify adjectives that are used identically (redundantly). In the present example, the first, third, and fourth adjectives are used in all three sorts, and each would be represented by 111 . Similarly, the second and fifth adjectives are used in the same pattern (110); as are the sixth, eighth, and tenth adjectives $(000)$; and seventh and ninth adjectives (100). The number of adjectives that are used in the same pattern corresponds to $n_{i}$ in the formula for calculating $H$.

4. After the patterns of use for all adjectives are compared, $\log _{2} n_{i}$ values are computed for each set of adjectives that are used similarly.

5 . When this process is completed for all adjectives, the $\log _{2} n_{i}$ values are summed, the quantity is multiplied by $(1 / n)$, and that quantity is subtracted from $\log _{2} n$. The resulting value, 1.97095 , is $H$.

$H$-Comp calculations were compared with those of two persons in order to test the accuracy of the program. Data from 40 participants were compared; in every case where there was a discrepancy, the manual calculation was in error.

\section{Program Requirements and Availability}

$H$-Comp runs on IBM PCs or compatibles under DOS. It has been found to run successfully on computers ranging from an IBM-XT clone to machines using a 486 processor, and on CGA and VGA video adapters. A copy of the program is available from the COMPsych PC software archive (Hornby \& Anderson, 1994) by anonymous ftp to gluon.hawk.plattsburgh.edu. Path = pub/compsych/ brmic. The program and source code can also be obtained from the author by sending a formatted disk and a self-addressed, postage-paid mailer.

\section{REFERENCES}

AtTNEave, F. (1959). Applications of information theory to psychology. New York: Holt.

Dixon, T. M., \& Baumeister, R. F. (1991). Escaping the self: The moderating effect of self-complexity. Personality \& Social Psychology Bulletin, 17, 363-368.
Hershberger, P. J. (1990). Self-complexity and health promotion: Promising but premature. Psychological Reports, 66, 1207-1216.

Hornby, P. A., \& ANDERson, M. D. (1994). COMPsych: The electronic software information service and PC software archive for psychology. Behavior Research Methods, Instruments, \& Computers, 26, 57-59.

Kalthoff, R. A., \& Niemeyer, R. A. (1993). Self-complexity and psychological distress: A test of the buffering model. International Journal of Personal Construct Psychology, 6, 327-349.

LINVILLE, P. W. (1982). The complexity-extremity effect and agebased stereotyping. Journal of Personality \& Social Psychology, 42, 193-211.

LINVILLE, P. W. (1985). Self-complexity and affective extremity: Don't put all of your eggs in one cognitive basket. Social Cognition, 3, 94-120.

LiNVILLE, P. W. (1987). Self-complexity as a cognitive buffer against stress-related illness and depression. Journal of Personality \& Social Psychology, 52, 663-676.

Linville, P. W., Fischer, G. W., \& SAlovey, P. (1986). Stereotyping and perceived distributions of social characteristics: An application to ingroup-outgroup perception. In J. F. Dovidio \& S. L. Gaertner (Eds.), Prejudice, discrimination, and racism (pp. 165-208). Orlando, FL: Academic Press.

LiNVILlE, P. W., \& JoNEs, E. E. (1980). Polarized appraisals of outgroup members. Journal of Personality \& Social Psychology, 38, 689-703.

Niedenthal, P. M., Setterlund, M. B., \& Wherry, M. B. (1992). Possible self-complexity and affective reactions to goal-relevant evaluation. Journal of Personality \& Social Psychology, 63, 5-16.

NiElsen, M. E. (1992). Domain-specific self-complexity: The intellectual and the religious. Unpublished doctoral dissertation, Northern Illinois University, DeKalb.

ScotT, W. A. (1969). The structure of natural cognitions. Journal of Personality \& Social Psychology, 12, 261-278.

SHOWERS, C. (1992). Compartmentalization of positive and negative self-knowledge: Keeping bad apples out of the bunch. Journal of Personality \& Social Psychology, 62, 1036-1049.

SMith, H. S., \& CoHEN, L. H. (1993). Self-complexity and reactions to a relationship breakup. Journal of Social \& Clinical Psychology, $12,367-384$.

\section{NOTE}

1. Linville has kindly offered to interested researchers a complete set of instructions, detailed recommendations for obtaining reliable data, and the adjective set used in her 1987 article. Contact P. Linville, Duke University, Fuqua School of Business, Durham, NC 27708.

(Manuscript received July 19, 1994; revision accepted for publication May 10, 1995.) 